\title{
(1) \\ CrossMark \\ A journey to the centre of the mediastinal lymph nodes
}

\author{
Beatriz Amat ${ }^{1}$ and Sandra Sapia ${ }^{2}$ \\ Affiliations: ${ }^{1}$ Pulmonology Dept, Hospital Universitario del Vinalopó, Elche, Spain. ${ }^{2}$ Pathology and Laboratory \\ Medicine, Weill Cornell Medical College in Qatar, Doha, Qatar. \\ Correspondence: Beatriz Amat, Hospital Universitario del Vinalopó, Pneumology, Tonico Sanano Mora 14, \\ Elche 03293, Spain. E-mail: beatrizamatahotmail.com
}

@ERSpublications

This study demonstrates the potential of EUS-guided nCLE. It is a novel approach that shows the best area for sampling a target lesion because it enables real-time visualisation of malignant infiltration in lung masses and lymph nodes. http://bit.ly/2JZYgWX

Cite this article as: Amat B, Sapia S. A journey to the centre of the mediastinal lymph nodes. Eur Respir J 2019; 53: 1900850 [https://doi.org/10.1183/13993003.00850-2019].

In 1864, Jules Verne wrote his famous book, Journey to the Center of the Earth, in which the discovery of an ancient manuscript found by Professor Lidenbrock proved that it was possible to travel to the bowels of the Earth. With this in mind, it would be worthwhile discussing the article published in this issue of the European Respiratory Journal, on the subject of needle-based confocal laser endomicroscopy (nCLE) [1].

Thanks to the birth of endoscopic ultrasound (EUS) in 2003, it is possible to travel to the centre of neoplastic lesions, regardless of them being tumour masses or metastatic lymph nodes. Nowadays, EUS is the standard technique for tissue sampling of a variety of masses, such as lung masses and mediastinal lymph nodes. However, after more than 15 years of performing EUS, it is now time that we had more information concerning those lesions in order to choose the optimal biopsy site. Furthermore, new techniques like confocal laser endomicroscopy (CLE) or elastography are now available. CLE is a relatively novel technique, which is slightly more than 10 years old $[2,3]$. It provides an in vivo histopathological assessment during the endoscopic process, as well as allowing for real-time image acquisition during the EUS process. However, for pneumological lesions, it is a rather new technique [4-6].

It is argued that the significant complication of bronchoscopy is not getting a diagnosis. This sentence was coined by S. Gasparini a few years ago during a lecture at the Hands-On-Workshop Thorakale Endoskopie in the Lungenklinik in Hemer (Germany), in January 2011. He also claimed that it is necessary to use the proper sampling instrument in the appropriate clinical setting. Otherwise, bronchoscopy may not be as optimal as it should be.

On our opinion, he was right, and this idea comes often to mind. In addition to that, nowadays, endobronchial ultrasound (EBUS) should also be included in the claim mentioned above. Secondly, it would be necessary to use the appropriate sampling instruments if they are available. Although there has been a revolution in our bronchoscopy rooms, owing to the increase in technological advancement [7], sometimes, we are still lacking some instruments, such as real-time equipment. These instruments would help us identify the most ideal and significant tumour area to get the most cost-effective puncture or biopsy.

It widely regarded that the growth pattern of neoplastic cells seems to be, somehow, at random; actually, that is not the case. It appears so for the sake of diagnostic purposes. In tumours, it is not just the cells of 
diagnostic interest that are present. Besides, the amount and arrangement of stromal components, necrosis, and even the remnants of the original tissues, collaborate with such heterogeneity. This "patchy" neoplastic growth hinders tumour sampling, as the access to cells with diagnostic value becomes difficult. That happens in both primary neoplasms and metastatic lymph nodes, and makes early diagnostics and treatment difficult.

In some cases, the lesion is rich in neoplastic cells and poor in stroma or other components, and adequate diagnostic yield can be obtained. However, malignant cells are often like planets in the universe: cell groups separated by abundant non-neoplastic elements that are of no value for diagnostic purposes. Alternatively, they are arranged focally like a discrete metastatic group in a lymph node. It could be said that when looking at the lymph node (as on a clock face) the tumor cells lie at 4 o'clock while the EBUS needle is tilted towards 7 or 8 o'clock. The results may be frustratingly negative or give poor yields since the needle could not appropriately reach the area of interest. In both examples, the use of CLE can provide high-resolution images leading to in vivo histological analysis and optical biopsies that could improve tumour sampling.

CLE is a laser-based technique that uses a fluorescent dye to stain the extracellular matrix and enables real-time visualisation of cell shapes and contours. One of the advantages of this technique is that it works as a real-time microscope that helps to select the optimal biopsy site. With adequate training, the operator should be able to identify the areas of interest for sampling, thus improving the diagnostic yield. CLE has also been found to be useful for the exploration of alveolar structures and mucosal tissue in vivo [4-6].

Currently, CLE can be performed using three different types of devices: endoscope-based (eCLE), probe-based (pCLE) and needle-based CLE (nCLE). In the latter, a CLE miniprobe passes through a 19-G EUS-FNA needle. However, to date, no publication uses EBUS needles because the CLE-probe does not fit through the 19-G EBUS needle. Nevertheless, as the authors point out, 19-G EBUS needles with larger inner diameters are expected to be available soon [1]. This fact is probably one of the reasons why the authors advocate for the use of the EUS 19-G needle.

Even though nCLE has already been described in peripheral pulmonary nodules [4], this paper is the first study with a significant number of patients for real-time identification of mediastinal lymph nodes or centrally located tumors using the nCLE technique. It is a proof based on principle study and shows that nCLE is a feasible and safe procedure. The authors were able to identify malignant cells in the majority (92\%) of patients with lung cancer. Therefore, the nCLE technique focuses on the optimal area for sampling, and it may, in the future, be used to select the best area for targeting a given treatment.

On the one hand, as the authors have acknowledged, nCLE also has some disadvantages in that it cannot replace traditional pathology, or rapid on-site cytopathology, assessment given that fluorescein does not stain cell nuclei. Likewise, on-site tumour marker assessment is not possible, either. Moreover, the authors provide no evidence that nCLE resulted in high diagnostic accuracy in comparison to the group not using nCLE. They cannot demonstrate that the use of nCLE made them change the puncture site. Thus, there needs to be more studies to demonstrate the benefit of the procedure objectively. On the other hand, this new field of confocal imaging allows definition and refinement of criteria to distinguish between benign or malignant lesions in correlation with the already consolidated histopathology criteria. The learning curve is also not well described as yet, and should include the need for the endoscopist to learn about cytopathology/pathology interpretation.

In conclusion, this study demonstrates the potential of EUS-guided nCLE. It is a novel approach that shows the ideal area for sampling a target lesion as it enables real-time visualisation of malignant infiltration in lung masses and lymph nodes. The integration of nCLE in navigation bronchoscopy is probably the next step, along with the development of the 19-G EBUS needle with an increased inner diameter. This improvement in our bronchoscopy practice opens an interesting debate: are the next-generation EBUS and robotic bronchoscopic navigational technology coming to us?

Acknowledgements: The authors would like to thank Dr García-Pachón for his disinterested help in the revision of this editorial, and also to our families for their constant support.

Conflict of interest: None declared.

\section{References}

1 Wijmans L, Yared J, de Bruin DM, et al. Needle-based confocal laser endomicroscopy for real-time diagnosing and staging of lung cancer. Eur Respir J 2019; 53: 1801520.

2 Becker V, Wallace MB, Fockens $\mathrm{P}$, et al. Needle-based confocal endomicroscopy for in vivo histology of intra-abdominal organs: first results in a porcine model (with videos). Gastrointest Endosc 2010; 71: 1260-1266. 
3 Meining A, Frimberger E, Becker V, et al. Detection of cholangiocarcinoma in vivo using miniprobe-based confocal fluorescence microscopy. Clin Gastroenterol Hepatol 2008; 6: 1057-1060.

4 Su Z, Zhong C, Li S, et al. Needle-based confocal laser endomicroscopy in the diagnosis of a peripheral pulmonary nodule: a preliminary report. J Thorac Dis 2017; 9: 2608-2612.

5 Hassan T, Piton N, Lachkar S, et al. A novel method for in vivo imaging of solitary lung nodules using navigational bronchoscopy and confocal laser microendoscopy. Lung 2015; 193: 773-778.

6 Fuchs FS, Zirlik S, Hildner K, et al. Confocal laser endomicroscopy for diagnosing lung cancer in vivo. Eur Respir J 2013; 41: 1401-1408.

7 Amat B. The increasing technology in bronchoscopy suites. Arch Bronconeumol 2010; 46: 285-287. 